\title{
Gastrointestinal tract modeling using organoids engineered with cellular and microbiota niches
}

\author{
Sungjin Min ${ }^{1}$, Suran $\mathrm{Kim}^{1}$ and Seung-Woo Cho ${ }^{1}$
}

\begin{abstract}
The recent emergence of organoid technology has attracted great attention in gastroenterology because the gastrointestinal (Gl) tract can be recapitulated in vitro using organoids, enabling disease modeling and mechanistic studies. However, to more precisely emulate the Gl microenvironment in vivo, several neighboring cell types and types of microbiota need to be integrated into Gl organoids. This article reviews the recent progress made in elucidating the crosstalk between $\mathrm{Gl}$ organoids and components of their microenvironment. We outline the effects of stromal cells (such as fibroblasts, neural cells, immune cells, and vascular cells) on the gastric and intestinal epithelia of organoids. Because of the important roles that microbiota play in the physiology and function of the Gl tract, we also highlight interactions between organoids and commensal, symbiotic, and pathogenic microorganisms and viruses. Gl organoid models that contain niche components will provide new insight into gastroenterological pathophysiology and disease mechanisms.
\end{abstract}

\section{Introduction}

The organoid is a miniaturized organ generated from stem cells grown in a dish. Various kinds of organoids can be produced depending on the target organs, such as the brain, liver, lung, kidney, stomach, and intestine ${ }^{1-6}$. Organoids contain various organ-specific cell types within a three-dimensional (3D) structure, thus containing the specific phenotype and physiology that each organ possesses. Organoids with these properties better mimic the in vivo environment than two-dimensional (2D) cell models, so they can be used for disease modeling and drug screening. In comparison with traditional animal models, organoids are cheaper and faster and are considered more ethical; further, their derivation from human stem cells enables better modeling of human physiology ${ }^{7}$. Moreover, organoids can be easily genetically manipulated, permitting the study of organogenesis and disease development ${ }^{8}$.

Correspondence: Seung-Woo Cho (seungwoocho@yonsei.ac.kr)

'Department of Biotechnology, Yonsei University, Seoul 03722, Republic of Korea

These authors contributed equally: Sungjin Min, Suran Kim
Despite the many advantages of organoids, there remain some limitations arising from scalability and reproducibility. Another major problem involves differences between organoids and actual organs due to the absence of surrounding tissue, such as the stromal cells that are present in connective tissue of any organ. Although the types of stromal cells are slightly different for each organ, they generally include fibroblasts, neural cells, immune cells and vascular cells. They are known to have a profound effect on an organ through immune responses, nutrient supply, paracrine signaling and extracellular matrix supply ${ }^{9-12}$. Thus, including these cell types during organoid culture is important for increasing the maturity and complexity of organoids ${ }^{13}$.

Organoid models of the gastrointestinal (GI) tract, intestinal and gastric organoids, were first developed in 2009 and 2010, respectively ${ }^{14,15}$. Researchers extracted $\mathrm{Lgr}^{+}$adult stem cells (ASCs) directly from the stomach and intestine and cultured them with the appropriate growth factors and supporting matrix. As described above, both of the resulting organoids consisted only of organ-specific tissue (gastric or intestinal epithelium), while other tissue types were excluded. ASC-derived GI

\section{(c) The Author(s) 2020}

(c) Open Access This article is licensed under a Creative Commons Attribution 4.0 International License, which permits use, sharing, adaptation, distribution and reproduction cc) in any medium or format, as long as you give appropriate credit to the original author(s) and the source, provide a link to the Creative Commons license, and indicate if changes were made. The images or other third party material in this article are included in the article's Creative Commons license, unless indicated otherwise in a credit line to the material. If material is not included in the article's Creative Commons license and your intended use is not permitted by statutory regulation or exceeds the permitted use, you will need to obtain permission directly from the copyright holder. To view a copy of this license, visit http://creativecommons.org/licenses/by/4.0/. 
organoids consist only of gastric epithelial cells such as parietal cells, chief cells, and surface pit cells or intestinal epithelial cells such as enterocytes, Paneth cells, and goblet cells. On the other hand, the generation of intestinal and gastric organoids from pluripotent stem cells (PSCs) was reported in 2011 and 2014, respectively, Since PSCs themselves have the capacity to differentiate into three germ layers (ectoderm, mesoderm, and endoderm), mesenchymal cells, including smooth muscle cells, fibroblasts, and myofibroblasts, are co-developed with PSC-derived GI organoids during differentiation. To date, the presence or absence of mesenchymal lineage cells has been known to be a main difference in the cell components of PSC-derived and ASC-derived organoids ${ }^{16,17}$. The presence of a small number of mesenchymal cells enables epithelial-mesenchymal interactions and eventually allows the successful differentiation of GI organoids ${ }^{18}$. However, the co-development of organoids with other environmental cells, such as neural cells, immune cells, and endothelial cells, has rarely been reported. This results in differences in the environment between the native organ and the organoid, which prevents the organoid from properly recapitulating the actual organ. To address this problem, many researchers are attempting to improve organoid culture using a variety of methods, such as three-dimensional (3D) bioprinting, biomaterials, and coculture $^{19,20}$. In particular, GI organoids are closely related to the microbiota. The organs of the GI tract have a mucosal surface on the lumen side, allowing various microbes to colonize there ${ }^{21}$. The microbes reside in the gut and influence intestinal biology, such as epithelial turnover, physiological processes and immune homeostasis, and they compete with other pathogens that enter through the diet and have a nonnegligible effect on the host $^{22,23}$. In fact, the intestinal microbiota affects drug pharmacokinetics and treatment outcomes, and its importance is becoming increasingly evident in drug discovery and clinical trials ${ }^{23,24}$. To increase the understanding of drug mechanisms, it seems necessary to incorporate microbiota into GI organoids. In addition to symbiosis with the host, some microbiota have a tremendous effect on severe GI tract disease. For example, Helicobacter pylori is the main cause of stomach diseases such as peptic ulcers, chronic gastritis, and gastric cancer, and intestinal microbes and viruses lead to diarrhea, inflammation, colitis, inflammatory bowel disease and even obesity ${ }^{25-27}$. The coculture system of microbiota and GI organoids would facilitate the study of GI tract diseases in terms of host-pathogen interactions ${ }^{28}$. Furthermore, due to the unique culture environment of specific microorganisms and viruses, organoids can be used as a platform to grow such species that previously have been difficult to maintain.
In this review, we introduce GI organoids integrated with cellular and microbiota niche components as tools for modeling the physiology and pathology of the GI tract (Fig. 1). First, we describe platforms involving the coculture of GI organoids with various stromal cells that reside in the native organ (Fig. 2). Then, we discuss how inoculation of GI organoids with microbiota can be used to investigate the pathophysiological effects of microorganisms and viruses on the GI tract (Fig. 2). These engineered GI organoids provide an effective alternative to conventional cell-based in vitro models and animal models for drug development and for studying GI diseases.

\section{Gastric organoids integrated with stromal cells}

The stomach is a complex organ containing various cell populations. It is comprised of the mucosa, submucosa, muscularis propria and serosa, which are organized into four closely connected layers. The gastric epithelium is divided into two main parts, the corpus (fundus) and antrum, which each contains different cell types. The epithelium of the corpus region contains many functional cells, including acid-secreting parietal cells and pepsinogen-secreting chief cells, while the epithelium of the antrum region consists mostly of mucus-producing cells. The lamina propria is a loose connective tissue under the gastric epithelium and contains many surrounding stromal cells, such as fibroblasts and immune cells. Nerves and blood vessels populate the submucosal region of the stomach, where they are thought to interact very closely with the gastric epithelium based upon their histological proximity ${ }^{29}$. However, the precise cellular interactions and their effects on the gastric epithelium remain unclear.

As mentioned, surrounding mesenchymal cells provide the gastric epithelium with a specific cellular niche in the stomach, but these cells do not exist in ASC-derived gastric organoids. To incorporate epithelial-mesenchymal interactions in organoids, immortalized stomach mesenchymal cells (ISMCs) were cocultured with gastric organoids $^{30}$. Coculture of organoids with ISMCs increased the number of all cellular components of the fundus (e.g., parietal cells, chief cells, surface pit cells, and mucous neck cells), which is a part of the stomach, and there was improved functionality of parietal cells ${ }^{30,31}$. Moreover, an integrated system comprised of glandular stomach cells and gastric mesenchymal myofibroblasts increased the differentiation and proliferation of gastric epithelium and supported their long-term maintenance ${ }^{32}$. These findings suggest that mesenchymal cells are important not only for gastric patterning during embryonic development but also for maintenance and functional activity of the gastric epithelium ${ }^{33,34}$. Wnt 


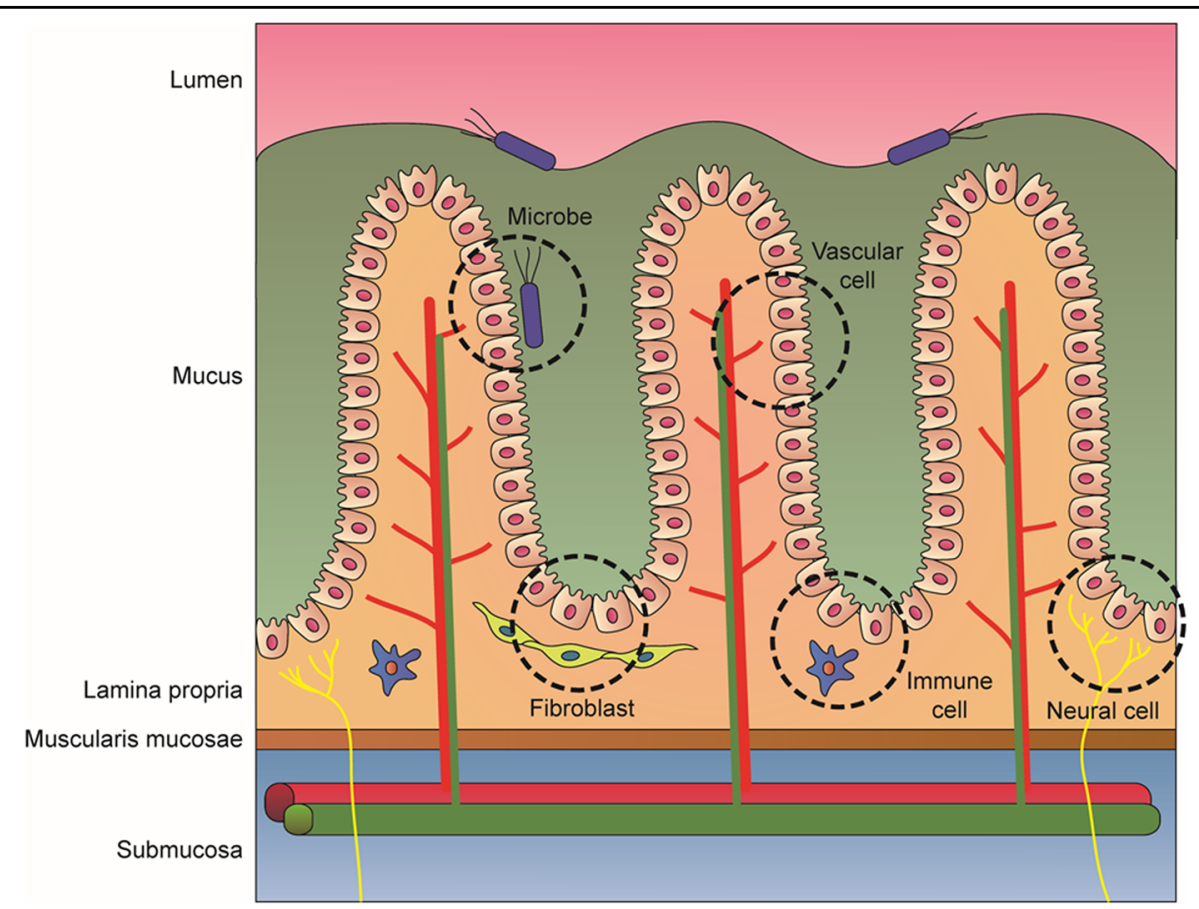

Fig. 1 Image of Gl epithelium and the surrounding tissues. In Gl tissues, there are numerous interactions between the epithelium and microbes or other cells, such as fibroblasts, vascular cells, immune cells and neural cells. Their communication has significant effects on the functions and homeostasis of the epithelium, indicating the necessity of including stromal cells and microbiota for producing functional 3D organoid models of the Gl tract.

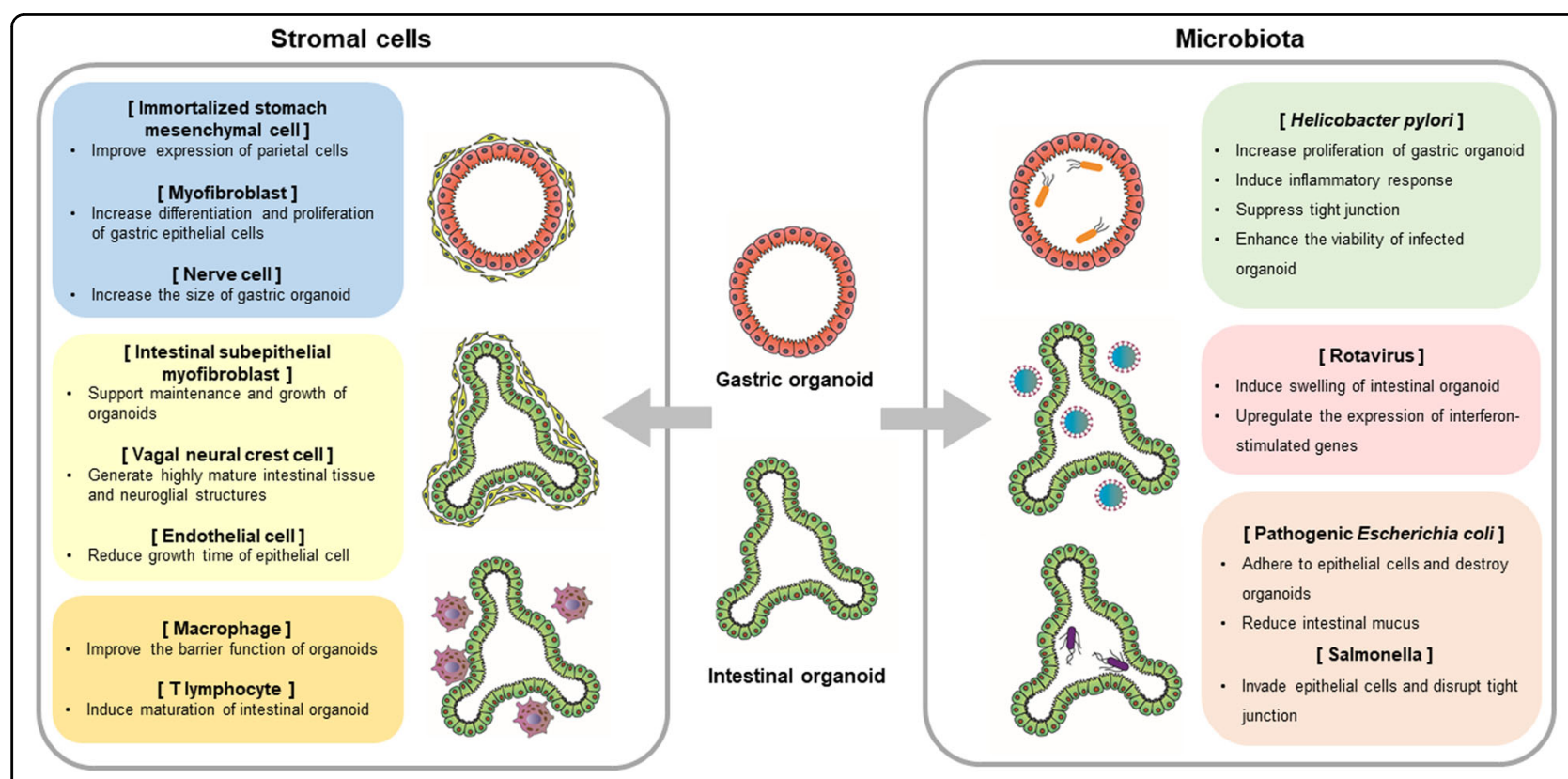

Fig. 2 Coculture models of Gl organoids with stromal cells and microbiota. The biological effects of various stromal cells and microbiota on $\mathrm{Gl}$ organoids in coculture models are described.

signaling is also known to be a crucial factor for the proliferation of gastric stem cells, and R-spondin (Rspo), which is provided by the surrounding stromal cells, enhances Wnt signaling. Consistent with this, organoid growth was shown to be supported in Rspo-free medium as long as the gastric organoids were cocultured with stromal cells, because endogenous Rspo was produced by stromal myofibroblasts ${ }^{35}$. This suggests that stromal 
myofibroblasts have a significant effect on the turnover and regeneration of the gastric epithelium.

The enteric nervous system (ENS), which interacts closely with the gastric epithelium, extends through the GI tract from the esophagus to the anus. The ENS is called a "second brain" because it interacts with the central nervous system (CNS) in both directions and can function without instructions from the brain and CNS. This autonomous system has significant effects on the motility, absorption of nutrients and hormone secretion of the digestive system ${ }^{36,37}$. Within the stomach, neurons of the ENS are primarily involved in gastric acid secretion and the release of histamine and 5 -hydroxytryptamine ${ }^{38}$. Coculture of gastric organoids with nerve cells derived from the myenteric plexus was found to enhance organoid growth, and this effect was attributed to Wnt signaling ${ }^{39}$. However, further studies utilizing such coculture models are required to understand the mechanism of interaction between the ENS and the gastric epithelium.

The stomach is protected from external infections and diseases by the barrier function of the gastric epithelium combined with an immense contribution of immune cells. The interaction between immune cells and the epithelium is closely linked to the gastric pathophysiology of bacterial infection, gastric cancer, and gastric repair ${ }^{40-42}$. Chakrabarti et al. established a model system for predicting the effect of immune checkpoint inhibitor therapy using a coculture system of gastric cancer organoids and immune cells $^{43}$. Expression of programmed death 1 (PD-1) in cytotoxic $\mathrm{T}$ lymphocytes (CTLs) was induced by gastric cancer organoids in the presence of dendritic cells (DCs), and the interaction of $\mathrm{PD}-1$ with programmed death ligand 1 (PD-L1) in gastric cancer cells inhibited tumor destruction. In organoid-based systems that provide coculture with immune cells or conditioned media of immune cells, pharmacological inhibition of the PD-L1 interaction with PD-1 was found to increase apoptosis of tumor cells. Although more research is required, gastric organoid models incorporating immune cells show promise as in vitro preclinical tools suitable for patientspecific immunotherapy.

\section{Gastric organoids infected with Helicobacter pylori}

$H$. pylori is the leading cause of fatal gastric diseases, including gastric cancer and peptic ulcer disease ${ }^{27,44}$. To study the pathogenesis of $H$. pylori and validate experimental drugs, an effective model system that can recapitulate the pathophysiology of the human stomach is needed. The 3D epithelial architecture of gastric organoids provides an in vitro model for infection studies that is superior to $2 \mathrm{D}$ cell cultures ${ }^{45}$, and they are more costeffective and time-effective than animal models. Infection studies utilizing gastric organoids have revealed numerous mechanistic insights. Upon microinjection of $H$. pylori into PSC-derived gastric organoids, the CagA protein of H. pylori was shown to bind to the c-Met receptor of organoid epithelial cells, leading to phosphorylation of c-Met and consequent activation of epithelial cell proliferation ${ }^{5}$. Although the effects of $H$. pylori on gastric epithelial cells, including increased proliferation and cell motility, have been reported in $2 \mathrm{D}$ conditions ${ }^{46,47}$, conventional $2 \mathrm{D}$ models lack diversity and polarity of epithelial cells, so they cannot accurately recapitulate the interactions between bacteria and host cells ${ }^{48}$. In contrast, microinjection of $H$. pylori into the cavity of 3D organoids with polarized epithelial structures can emulate physiological changes and pathological events following $H$. pylori infection in the stomach better than $2 \mathrm{D}$ epithelial cell models with $H$. pylori simply added to the culture medium. Microinjection of $H$. pylori into ASCderived human gastric organoids also confirmed that $H$. pylori activates the NF- $\mathrm{kB}$ pathway and chemokine IL8 , which is a target of NF- $\mathrm{kB}$, resulting in an inflammatory response $^{49}$. Boccellato et al. successfully generated a polarized gastric epithelial layer from a gastric organoid using an air-liquid interface and confirmed that all phenotypes seen in the antral region of the stomach, including mucus production, are observed in this layer ${ }^{50}$. Investigating the distinct responses of the gastric epithelium to $H$. pylori using this model system, it was also found that undifferentiated basal cells had a stronger inflammatory response than foveolar cells.

The organoid model system can be used to validate established hypotheses and discover new mechanisms of H. pylori pathology. For example, it has been known that H. pylori promotes proliferation of the gastric epithelium, but the detailed mechanism behind this occurrence is unclear. Using gastric organoids, Bertaux-Skeirik et al. showed that CD44, which was already known to be associated with c-Met, has significant involvement in $t$ he epithelial proliferation caused by CagA-positive H. pylori $^{48}$. They also confirmed that atrophic gastritis caused by $H$. pylori was repressed by treatment with a CD44 peptide inhibitor. Another study found that the proliferation of gastric organoids infected with $H$. pylori is regulated by CagA and $\beta$-catenin signaling, and mislocalization and suppression of the cancer-associated tight junction protein claudin- 7 is induced by $\beta$-catenin and snail activation ${ }^{51}$; these data increase our understanding of the mechanism by which $H$. pylori causes gastric cancer. Huang et al. also used gastric organoids to study how $H$. pylori is attracted to and colonizes the gastric epithelium $^{52}$. They revealed that the H. pylori chemoreceptor $\mathrm{TlpB}$ recognizes urea, a metabolite produced by gastric organoids, causing $H$. pylori to be rapidly attracted to the epithelium, where it can modify the gastric microenvironment by hydrolyzing urea with urease. 
To account for the important role of the immune response in disease, immune cells have been introduced into organoids. H. pylori infection was shown to increase the expression of PD-L1 in gastric epithelial cells via the Sonic hedgehog (Shh) signaling pathway ${ }^{53}$. Based on the fact that PD-L1 expression is associated with the immune system $^{54}$, DCs and CTLs were cocultured with gastric organoids to study the interaction between PD-L1 and PD-1 in an infected gastric epithelium ${ }^{53}$. The increased expression of PD-L1 in $H$. pylori-infected organoids increased organoid viability through the interaction of PD-L1 with PD-1 that was expressed by CTLs, while pharmacological inhibition of PD-1 blocked this interaction, inducing death of infected epithelial cells. In a mouse model of infection, DCs were recruited to the gastric epithelium, where they interacted directly with $H$. pylori $^{55}$. Similar findings were recapitulated using a gastric organoid model, where monocyte-derived DCs were found to migrate to and interact with uninfected gastric epithelial organoids, and their migration was increased during $H$. pylori infection ${ }^{56}$. This augmented recruitment of DCs was shown to be caused by multiple chemokines derived from infected gastric organoids, while the recruited DCs played a role in the phagocytosis of $H$. pylori, similar to what occurs in gastric mucosa in vivo. As such, gastric organoids have proven to be an excellent in vitro infection model that can overcome the limitations of existing model systems, and they are expected to accelerate the development of etiological research.

\section{Intestinal organoids integrated with stromal cells}

The intestinal epithelium is the innermost part of the mucosa layer surrounding the intestinal lumen, where direct encounters with nutrients and foreign substances entering the GI tract occur. It is composed of specific intestinal cells that play important roles in digestive, absorptive, protective and secretory functions. The intestinal lamina propria, located beneath the epithelium, comprises various types of mesenchymal cells, such as fibroblasts, myofibroblasts, mural cells, immune cells, and smooth muscle cells of the muscularis mucosae ${ }^{57,58}$. Dense networks of blood vessels and nerves extend throughout the intestinal layers, assisting with the diverse functions of the intestine ${ }^{59-61}$. However, ASC-derived intestinal organoids, which recapitulate only the intestinal epithelium, and PSC-derived intestinal organoids, which mimic intestinal epithelium and some mesenchymal cells, lack these complex intercellular interactions. Thus, similar to gastric organoid models, the coculture of intestinal organoids with multiple cell types has been implemented to better simulate the in vivo intestinal cellular niche ${ }^{19,62}$.

Intestinal subepithelial myofibroblasts (ISEMFs) provide pivotal cues regarding the intestinal stem cell niche by secreting growth factors such as Wnt ligands and BMP antagonists ${ }^{57,63,64}$. Coculture of ASC-derived intestinal organoids with ISEMFs permitted long-term culture of organoids in the absence of some growth factors necessary for organoid culture ${ }^{65}$. The presence of ISEMFs also promoted the formation of larger and more complex intestinal organoids than what was observed in monocultured organoids, and the conditioned medium of ISEMFs also promoted intestinal organoid growth ${ }^{66}$. These organoids cocultured with ISEMFs were exhibited more successful engraftment and proliferation upon transplantation. Coculture with ISEMFs also aided the expansion of mouse intestinal organoids, even in collagen gels $^{67}$. In the case of human intestinal organoids, monocultured organoids seeded in collagen gels underwent autolysis within 2-3 days; however, coculture with ISEMFs or supplementation with the conditioned medium from ISEMFs enabled rapid growth while maintaining a 3D structure in the collagen gel ${ }^{68}$. In an organotypic cell culture system consisting of an additional acellular layer and a layer with cells on a Transwell $^{\circ}$ permeable support system, intestinal organoids cocultured with intestinal myofibroblasts also showed increases in diameters and improved viability ${ }^{39}$. These results indicate that ISEMFs provide useful niche factors for intestinal stem cells and for the growth of intestinal organoids.

The ENS is an indispensable element involved in the manipulation of various intestinal functions, such as peristalsis, coordination of blood flow, regulation of the intestinal epithelial barrier and secretion of fluid and electrolytes ${ }^{36,69,70}$. However, both ASC-derived intestinal organoids and PSC-derived intestinal organoids lack ENS direction. To address this shortcoming, efforts have been undertaken to differentiate PSCs into vagal neural crest cells (NCCs), from which the ENS is mainly derived, and integrate them into organoids ${ }^{71,72}$. Fattahi et al. established a method for neural differentiation and maturation of enteric neural crest (ENC) precursors and confirmed the in vivo interactions of the precursor cells with mouse primary intestinal tissue using a tissue engineering approach $^{71}$. Organoid units extracted from mouse intestines were directly and simultaneously seeded with human PSC-derived ENCs on a polyglycolic acid scaffold, and after implantation, they formed an intestine-like structure that expressed human neural cell markers in both the epithelial and muscle layers. Workman et al. generated intestinal organoids with functional neurons and glia by mechanical aggregation of PSC-derived $\mathrm{mid} /$ hind spheroids with PSC-derived NCCs; following transplantation into mice, these organoids developed into highly mature intestinal tissue with neuroglial structures similar to those of a myenteric and submucosal plexus ${ }^{72}$. In addition, organoids integrated with NCCs derived from PSCs with PHOX2B mutations, which are associated with complete aganglionosis of the bowel, grew poorly and showed a 
pattern of suppressed smooth muscle development. These findings indicate that NCCs have noncellular autonomous effects on the development of intestinal cells and that these engineered organoids can be used as a specific in vitro model of Hirschsprung's disease. It has also been shown that PSC-derived intestinal organoids integrated with neural and muscle cells can be generated by differentiating the PSCs into the three germ layers using a strategy that mimics in vivo organogenesis ${ }^{73}$. These integrated intestinal organoids expressed the enteric neural marker known as protein gene product 9.5 (PGP9.5), had neurofilaments distributed throughout the mesenchymal area and exhibited contractile movement that could be controlled by treatment with histamine and atropine.

The ability to mimic the intestinal immune system provides another important objective for organoid models. Since the intestinal lumen is continuously in contact with foreign materials and enteric commensal microbes, a defense system is essential for protecting the body from invasion. The intestine has special structures called gutassociated lymphoid tissues (GALTs) that contain specialized $M$ cells, which serve as an inductive site for the mucosal immune response; GALTs also contain many different immune cells, such as macrophages, DCs, $\mathrm{T}$ cells, B cells, plasma cells and mast cells, which play a key role in immune homeostasis ${ }^{7-77}$. To facilitate the interaction between intestinal epithelial cells and immune cells, Noel et al. seeded intestinal stem cell-derived organoids on a Transwell ${ }^{\oplus}$ permeable support to generate epithelial monolayers that were cocultured with monocyte-derived macrophages ${ }^{78}$. The presence of macrophages enhanced the height and transepithelial electrical resistance of epithelial cells, suggesting that they have positive effects on the maturation and barrier functions of the intestinal epithelium. Upon infection with pathogenic Escherichia coli (E. coli) to mimic in vivo luminal exposure, the cocultured macrophages sensed $E$. coli and extended toward the epithelium. In addition, the number of viable $E$. coli was reduced, and the breakdown of the epithelial barrier was partially protected compared with that of the epithelial monoculture model. This coculture model provides insight into host-pathogen interactions and the innate immune response in the intestinal mucosa.

Unlike the above method of interrogating the direct effects of immune cells on intestinal organoids, some studies have investigated the indirect effects of immune cells on intestinal organoids using interleukins. ASCderived intestinal organoids that were cocultured with lymphoid cells, which produce interleukin-22 (IL-22), were significantly larger than monocultured organoids ${ }^{79}$. Similarly, when recombinant IL-22 was added to the culture medium, an improvement in intestinal organoid growth was also found. This indicated that the immune system is a component of the intestinal stem cell niche and regulates intestinal regeneration. In a PSC-derived intestinal organoid system, coculture with $\mathrm{T}$ lymphocytes and treatment with interleukin-2 (IL-2) led to in vitro maturation of intestinal organoids that retained fetal intestinal properties ${ }^{80}$. Jung et al. confirmed that IL-2, the most abundant cytokine secreted by $\mathrm{T}$ lymphocytes, produces PSC-derived intestinal organoids with a gene expression profile and functionality that is similar to that of adult intestinal tissue ${ }^{80}$. These results indicate that soluble factors secreted by immune cells have a profound effect on the regeneration and maturation of intestinal tissue and that inclusion of immune cell interactions is an effective strategy for overcoming the limitations of functionally immature PSC-derived intestinal organoids.

Although blood vessels are crucial for intestinal function, the introduction of vasculature into intestinal organoids has rarely been attempted. Kasendra et al. used microfluidic devices comprised of two parallel microchannels separated by a porous polydimethylsiloxane (PDMS) membrane to combine the intestinal epithelium and the microvascular endothelium in vitro ${ }^{81}$. To generate a vascularized intestine-on-a-chip, intestinal epithelial cells dissociated from organoids were seeded on the top of the PDMS membrane, and intestinal microvascular endothelial cells were plated underneath the PDMS membrane. The presence of endothelial cells helped to shorten the growth time of the intestinal epithelial cells, and this vascularized intestine unit in the chip also exhibited genetic profiles similar to those of the in vivo duodenum. While they used microfluidic devices to attract vessel networks to the intestinal organoid, other researchers used in vivo transplantation to induce spontaneous vascularization. Watson et al. implanted intestinal organoids differentiated from human PSCs into a mouse kidney, which is a highly vascularized tissue ${ }^{82}$. These transplanted organoids not only became more mature but also had blood vessels that extended from the host tissue.

\section{Intestinal organoids that incorporate microbiota and viruses}

The intestine is a complex ecosystem composed of myriad coexisting bacteria, fungi and viruses that influence GI physiology ${ }^{83,84}$. The intestinal microbiota defends against the growth of invading bacteria and breaks down food to assist nutrient absorption by intestinal epithelial cells. They also produce metabolites to regulate immunity and other physiological activities. Therefore, when the balance of the intestinal ecosystem is perturbed, various diseases, such as inflammatory bowel disease and obesity, can occur ${ }^{85}$. Because the intestinal microbiota plays a role as a third organ ${ }^{86}$, interest in it among the research community, as well as the food industry is rapidly 
increasing. To meet these demands, several studies of intestinal microbial interactions have been conducted in an in vitro environment using intestinal organoids.

Some researchers have studied the effects of coculture with intestinal organoids and commensal bacteria such as Lactobacillus, a probiotic species that enhances the barrier function of the intestinal epithelium. Coculture with Lactobacillus rhamnosus increased organoid proliferation and differentiation into Paneth cells ${ }^{87}$, while Lactobacillus reuteri D8 improved the growth of intestinal organoids and protected against damage caused by the proinflammatory cytokine TNF- $\alpha^{88}$. In addition, Hill et al. microinjected the nonpathogenic Escherichia coli strain ECOR2 into the lumen of PSC-derived intestinal organoids to establish an in vitro model for exploring hostbacterial symbiotic interactions in the neonatal intestine $^{89}$. After microinjection, ECOR2 grew rapidly inside the lumen, and the organoid was maintained and did not become disrupted, confirming that both organisms were being stably cocultured. Interestingly, the stimuli by bacterial contact increased barrier function and antimicrobial defense and enhanced tissue maturation. These organoid-based microbial studies provided a foundation for revealing the mechanism by which probiotics contribute to human health.

In addition to commensal bacteria, pathogenic bacteria Escherichia coli ${ }^{90-93}$, Salmonella ${ }^{94-96}$, Clostridium diffcile $^{97}$, and Cryptosporidium ${ }^{98}$ can be incorporated into intestinal organoid systems to understand the effects of bacterial pathogens on the intestinal epithelium. Colonization of enterohemorrhagic E. coli (EHEC) in a human colonic organoid monolayer decreased intestinal mucus and destroyed microvilli, facilitating bacterial access to and infection of epithelial cells ${ }^{93}$. The mechanism was found to involve a reduction of brush border resident protein protocadherin 24 (PCDH24) by the EHEC protease EspP, demonstrating the ability of the model system to uncover the molecular mechanisms responsible for early intestinal colonization. The addition of Salmonella enterica serovar Typhimurium to organoid culture medium diminished intestinal epithelial tight junctions and led to bacterial invasion, with infected organoids exhibiting pathophysiological features such as NF- $\mathrm{kB}$ pathway activation and stem cell reduction ${ }^{94}$. To establish an in vitro human model for Clostridium difficile infection or Cryptosporidium parvum infection, direct contact between the lumen of intestinal organoids and bacteria was achieved using a microinjection technique $^{97,98}$. Clostridium difficile persisted in the organoids and produced toxins TcdA and TcdB, which have the ability to destroy the intestinal epithelium ${ }^{97}$. Cryptosporidium parvum propagated inside the organoid and completed its entire life cycle, which is only possible inside a suitable host ${ }^{98}$.
Enteric viruses are also major disease agents that easily enter the intestine through water or food. Their mechanisms of propagation and replication were impossible to study in vitro prior to the development of organoids. Rotavirus and norovirus are the most common viruses that cause GI disease. Finkbeiner et al. observed that intestinal organoids supported the replication of rotavirus isolated directly from stool samples, despite this virus being previously difficult or impossible to culture ${ }^{99}$. Moreover, rotavirus was found to infect not only intestinal epithelial cells but also adjacent mesenchymal cells ${ }^{99}$, providing a new perspective on rotavirus pathophysiology. Rotavirus could also replicate in organoids derived from three different regions of the small intestine, all of which recapitulated in vivo characteristics, such as host susceptibility and preferential infection of differentiated cells $^{100}$. Organoids have been used to construct personalized infection models with rotavirus derived from individual patients as a way to evaluate patient-specific antiviral therapy ${ }^{101}$. Although human norovirus (HNV) has been difficult to culture in transformed epithelial cell lines and animal models, an intestinal organoid-derived monolayer supported a productive and complete HNV replication cycle, and diverse HNV strains were able to replicate in this system ${ }^{102}$.

Intestinal organoids offer a superior in vitro model system for the cultivation of microbiota that influence GI physiology and for understanding how they encounter the intestinal epithelium and cause disease. Mechanistic details obtained from such modeling may provide new avenues for the prevention and treatment of many GI tract diseases.

\section{Conclusions and outlook}

Organoid models are increasingly being used to explore biological phenomena associated with tissue development and differentiation, as well as human diseases. GI organoid-based integrated systems provide in vitro models that more accurately mimic the in vivo environment of the GI tract than previous culture systems, and they lay the foundation for deeper insights into interactions of the GI epithelium with cellular/microbiota niches. Several studies have utilized engineered organoid systems to elucidate how surrounding cells or microbiota affect GI pathophysiology. However, current techniques with multiple cocultures and strategies for the reconstitution of culture environments need to be further improved to enhance the structural complexity and functional maturation of engineered organoids. Current methods of GI organoid coculture are mostly performed by simple mixing components, which fails to recapitulate the regularly arranged structures observed in vivo. Moreover, there is a need for the development of new culture systems that can individually supply different culture media 


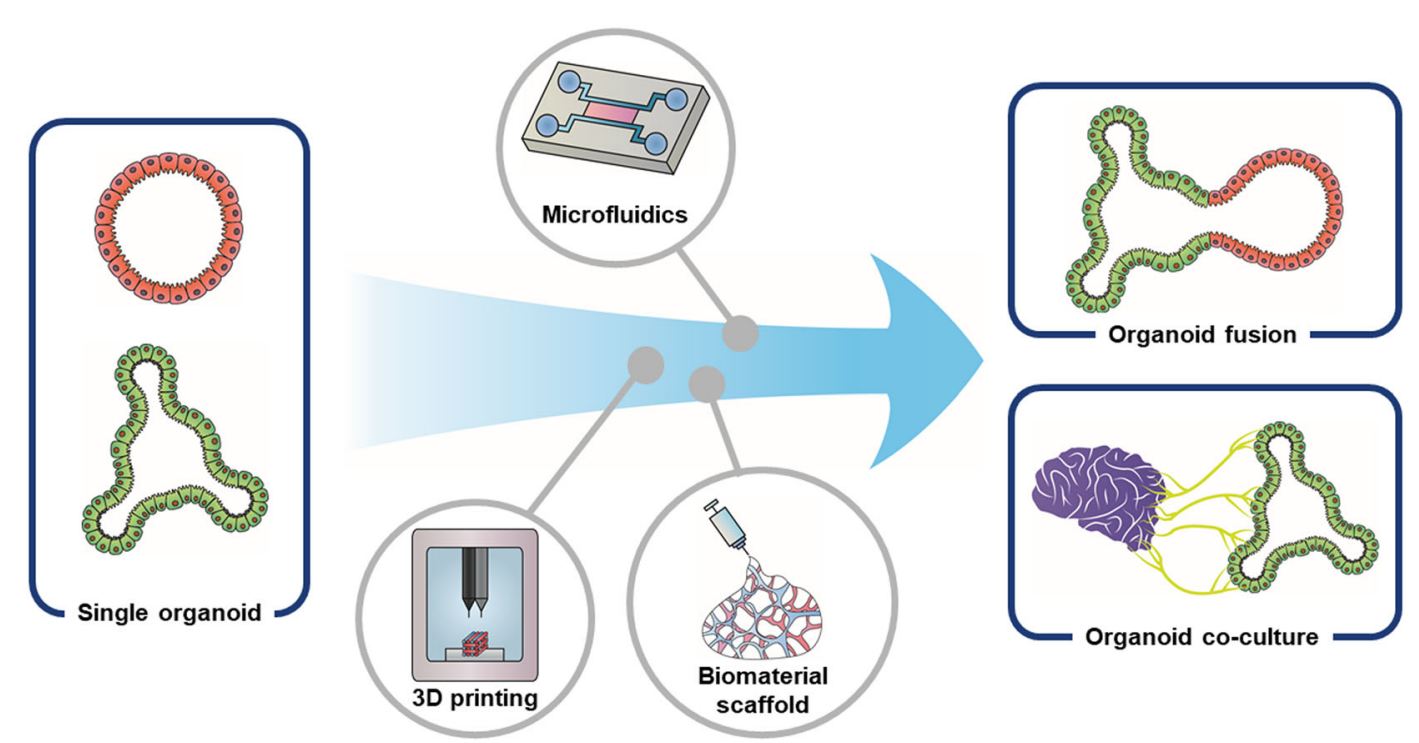

Fig. 3 Bioengineered platforms for developing Gl organoids assembled with other types of organoids. Through bioengineering techniques such as 3D bioprinting and the use of microfluidics and biomaterials, a novel integrated $\mathrm{Gl}$ system can be developed by connecting the various organoids that make up the digestive system or by coculturing with completely different types of organoids such as brain organoids. These innovative organoid systems enable the modeling of highly complex human physiology, such as reciprocal regulation between the Gl tract and other major organs, and pathological phenomena associated with diseases.

that are suitable for different cell types. The focus must be not only on surrounding cells and microbiota but also on the surrounding extracellular matrix $(\mathrm{ECM})$ because most research to date has utilized Matrigel ${ }^{\oplus}$, which does not provide a tissue-specific environment. Various technologies are being developed for this purpose, such as 3D bioprinting and the use of microfluidic devices and biomaterials ${ }^{103-108}$. Bioprinting has the capacity to encapsulate various cells in a compatible hydrogel and to place them in the desired position suitable for coculture. This is an automated and efficient system that can achieve tissue complexity by incorporating a complex network of vasculature and innervation ${ }^{103,109}$. Microfluidic devices can be fabricated such that different cells can be seeded in different zones, making these devices ideal for coculture $^{110}$. For example, angiogenesis can be mimicked by placing organoids and vascular cells in different locations and connecting them using soluble factors ${ }^{104}$. Microfluidic devices are also well suited for modeling because of their ability to provide fluid flow that is similar to what is observed in vivo. The coupling of a microfluidic device with an organoid has recently been termed "organoid-ona-chip", and this represents an innovative approach in biomedical research ${ }^{105,106}$. Moreover, various engineered materials for organoid culture containing a decellularized matrix and a chemically defined matrix are being actively developed $^{106,107,111}$.

Methods employing the fusion of organoids and coculture of multiple organoids have recently emerged
(Fig. 3) ${ }^{112-114}$. A multiorgan integrated system fusing three different types of digestive system organoids on a microfluidic device has been used to identify crosstalk between organoids ${ }^{113}$. A new protocol for generating integral multiorgan structures of the digestive system has also been reported, leading to the fusion of two PSCderived spheroids that correspond to the anterior and posterior gut, as well as to the creation of hepato-biliarypancreatic organoids that establish boundary interactions between two spheroids without any extrinsic factors ${ }^{114}$.

Drug screening with only one type of organoid can be a limiting factor because the in vivo degradation and absorption of a drug occurs through various digestive organs. In particular, the gut and liver communicate with each other through portal veins and biliary tracts, enabling microbial products such as microbial-associated molecular patterns (MAMPs) to travel from the gut to the liver, causing inflammation and liver damage ${ }^{115}$. Liver diseases such as nonalcoholic fatty liver disease (NAFLD), alcoholic liver disease (ALD), cirrhosis, and hepatocellular carcinoma (HCC) are also reported to be associated with changes in the gut microbiome. The development of an integrated system consisting of several digestive organoids and microbiota makes it possible to screen drugs under conditions that mimic an in vivo environment.

Gut-brain crosstalk is closely related to homeostasis of the GI tract and cognitive ability of the brain ${ }^{116}$. In particular, autism spectrum disorder (ASD), cerebral palsy $(\mathrm{CP})$, and spina bifida, which are neurodevelopmental 
diseases, have been reported to be associated with GI abnormalities ${ }^{117,118}$. Therefore, a novel model system created by the coculture of GI organoids with brain organoids could be used to study the mechanisms underpinning these disorders. Likewise, the development of systems integrating various other organoids may elucidate many other physiological and pathological conditions throughout the body.

\section{Acknowledgements}

This work was supported by grants (2015M3A9B4071076 and 2018M3A9H1021382) from the National Research Foundation of Korea (NRF) funded by the Ministry of Science and ICT (MSIT), Republic of Korea.

\section{Conflict of interest}

The authors declare that they have no conflict of interest.

\section{Publisher's note}

Springer Nature remains neutral with regard to jurisdictional claims in published maps and institutional affiliations.

Received: 31 October 2019 Revised: 21 December 2019 Accepted: 27 December 2019.

Published online: 26 February 2020

\section{References}

1. Lancaster, M. A. et al. Cerebral organoids model human brain development and microcephaly. Nature 501, 373-379 (2013).

2. Takebe, $T$. et al. Vascularized and functional human liver from an IPSCderived organ bud transplant. Nature 499, 481-484 (2013).

3. Dye, B. R. et al. In vitro generation of human pluripotent stem cell derived lung organoids. Elife 4, https://doi.org/10.7554/eLife.05098 (2015).

4. Taguchi, A. et al. Redefining the in vivo origin of metanephric nephron progenitors enables generation of complex kidney structures from pluripotent stem cells. Cell Stem Cell 14, 53-67 (2014).

5. McCracken, K. W. et al. Modelling human development and disease in pluripotent stem-cell-derived gastric organoids. Nature 516, 400-404 (2014).

6. Spence, J. R. et al. Directed differentiation of human pluripotent stem cells into intestinal tissue in vitro. Nature 470, 105-109 (2011).

7. Lehmann, R. et al. Human organoids: a new dimension in cell biology. Mol. Biol. Cell 30, 1129-1137 (2019).

8. Fujii, M., Clevers, H. \& Sato, T. Modeling human digestive diseases with CRISPR-Cas9-modified organoids. Gastroenterology 156, 562-576 (2019).

9. Nowarski, R., Jackson, R. \& Flavell, R. A. The stromal intervention: regulation of immunity and inflammation at the epithelial-mesenchymal barrier. Cell $\mathbf{1 6 8}$ 362-375 (2017).

10. Daniel, E. \& Cleaver, O. Vascularizing organogenesis: lessons from developmental biology and implications for regenerative medicine. Curr. Top. Dev. Biol. 132, 177-220 (2019).

11. Bani, D. \& Nistri, S. New insights into the morphogenic role of stromal cells and their relevance for regenerative medicine. lessons from the heart. J. Cell Mol. Med. 18, 363-370 (2014).

12. Kolonin, M. G., Evans, K. W., Mani, S. A. \& Gomer, R. H. Alternative origins of stroma in normal organs and disease. Stem Cell Res. 8, 312-323 (2012).

13. Takebe, T. \& Wells, J. M. Organoids by design. Science 364, 956-959 (2019).

14. Sato, T. et al. Single Lgr5 stem cells build crypt-villus structures in vitro without a mesenchymal niche. Nature 459, 262-265 (2009).

15. Barker, N. et al. Lgr5(+ve) stem cells drive self-renewal in the stomach and build long-lived gastric units in vitro. Cell Stem Cell 6, 25-36 (2010).

16. Yin, Y. \& Zhou, D. Organoid and enteroid modeling of Salmonella infection. Front Cell Infect. Microbiol 8, 102 (2018).

17. Brassard, J. A. \& Lutolf, M. P. Engineering stem cell self-organization to build better organoids. Cell Stem Cell 24, 860-876 (2019).

18. McCauley, H. A. \& Wells, J. M. Pluripotent stem cell-derived organoids: using principles of developmental biology to grow human tissues in a dish. Development 144, 958-962 (2017).
19. Holloway, E. M., Capeling, M. M. \& Spence, J. R. Biologically inspired approaches to enhance human organoid complexity. Development 146, https://doi.org/10.1242/dev.166173 (2019).

20. Kim, S., Cho, A. N., Min, S., Kim, S. \& Cho, S. W. Organoids for advanced therapeutics and disease models. Adv. Therapeutics 2, 1800087 (2019).

21. Hao, W. L. \& Lee, Y. K. Microflora of the gastrointestinal tract: a review. Methods Mol. Biol. 268, 491-502 (2004).

22. Parker, A., Lawson, M. A. E., Vaux, L. \& Pin, C. Host-microbe interaction in the gastrointestinal tract. Environ. Microbiol 20, 2337-2353 (2018).

23. Swanson, H. I. Drug metabolism by the host and gut microbiota: a partnership or rivalry? Drug Metab. Dispos. 43, 1499-1504 (2015).

24. Enright, E. F., Gahan, C. G., Joyce, S. A. \& Griffin, B. T. The impact of the gut microbiota on drug metabolism and clinical outcome. Yale J. Biol. Med. $\mathbf{8 9}$ 375-382 (2016).

25. Baumler, A. J. \& Sperandio, V. Interactions between the microbiota and pathogenic bacteria in the gut. Nature $\mathbf{5 3 5}, 85-93$ (2016).

26. Turnbaugh, P. J. et al. An obesity-associated gut microbiome with increased capacity for energy harvest. Nature 444, 1027-1031 (2006).

27. Hooi, J. K. et al. Global prevalence of Helicobacter pylori infection: systematic review and meta-analysis. Gastroenterology 153, 420-429 (2017).

28. Bartfeld, S. Modeling infectious diseases and host-microbe interactions in gastrointestinal organoids. Dev. Biol. 420, 262-270 (2016).

29. Sobala, G. M. et al. Acute Helicobacter pylori infection: clinical features, local and systemic immune response, gastric mucosal histology, and gastric juice ascorbic acid concentrations. Gut 32, 1415-1418 (1991).

30. Schumacher, M. A. et al. The use of murine-derived fundic organoids in studies of gastric physiology. J. Physiol. 593, 1809-1827 (2015).

31. Kim, B. M., Buchner, G., Miletich, I., Sharpe, P. T. \& Shivdasani, R. A. The stomach mesenchymal transcription factor Barx1 specifies gastric epithelial identity through inhibition of transient Wnt signaling. Dev. Cell 8, 611-622 (2005).

32. Katano, T. et al. Gastric mesenchymal myofibroblasts maintain stem cell activity and proliferation of murine gastric epithelium in vitro. Am. J. Pathol. 185, 798-807 (2015).

33. Fukamachi, H., Mizuno, T. \& Takayama, S. Epithelial-mesenchymal interactions in differentiation of stomach epithelium in fetal mice. Anat. Embryol. 157, 151-160 (1979).

34. McCracken, K. W. \& Wells, J. M. Mechanisms of embryonic stomach development. Semin Cell Dev. Biol. 66, 36-42 (2017).

35. Sigal, M. et al. Stromal R-spondin orchestrates gastric epithelial stem cells and gland homeostasis. Nature 548, 451 (2017).

36. Furness, J. B. The enteric nervous system and neurogastroenterology. Nat. Rev. Gastroenterol. Hepatol. 9, 286-294 (2012).

37. Rao, M. \& Gershon, M. D. The bowel and beyond: the enteric nervous system in neurological disorders. Nat. Rev. Gastroenterol. Hepatol. 13, 517-528 (2016).

38. Mayer, E. A. Gut feelings: the emerging biology of gut-brain communication. Nat. Rev. Neurosci. 12, 453 (2011).

39. Pastula, A. et al. Three-dimensional gastrointestinal organoid culture in combination with nerves or fibroblasts: a method to characterize the gastrointestinal stem cell niche. Stem Cells Int. 2016, 3710836 (2016).

40. Roth, K. A., Kapadia, S. B., Martin, S. M. \& Lorenz, R. G. Cellular immune responses are essential for the development of Helicobacter felis-associated gastric pathology. J. Immunol. 163, 1490-1497 (1999).

41. Tu, S. et al. Overexpression of interleukin-1 beta induces gastric inflammation and cancer and mobilizes myeloid-derived suppressor cells in mice. Cancer Cell 14, 408-419 (2008).

42. Tarnawski, A. S. Cellular and molecular mechanisms of gastrointestinal ulcer healing. Digestive Dis. Sci. 50, S24-S33 (2005).

43. Chakrabarti, J. et al. Hedgehog signaling induces PD-L1 expression and tumor cell proliferation in gastric cancer. Oncotarget 9, 37439 (2018).

44. Peery, A. F. et al. Burden of gastrointestinal, liver, and pancreatic diseases in the United States. Gastroenterology 149, 1731-1741.e1733 (2015).

45. Pompaiah, M. \& Bartfeld, S. in Molecular Pathogenesis and Signal Transduction by Helicobacter pylori 149-168 (Springer, 2017).

46. Fan, X. G., Kelleher, D., Fan, X. J., Xia, H. X. \& Keeling, P. W. Helicobacter pylori increases proliferation of gastric epithelial cells. Gut 38, 19-22 (1996).

47. Churin, Y. et al. Helicobacter pylori CagA protein targets the c-Met receptor and enhances the motogenic response. J. Cell Biol. 161, 249-255 (2003).

48. Bertaux-Skeirik, N. et al. CD44 plays a functional role in Helicobacter pyloriinduced epithelial cell proliferation. PLoS Pathog. 11, e1004663 (2015). 
49. Bartfeld, S. et al. In vitro expansion of human gastric epithelial stem cells and their responses to bacterial infection. Gastroenterology 148, 126-136.e126 (2015).

50. Boccellato, F. et al. Polarised epithelial monolayers of the gastric mucosa reveal insights into mucosal homeostasis and defence against infection. Gut 68, 400-413 (2019)

51. Wroblewski, L. E. et al. Helicobacter pylori targets cancer-associated apicaljunctional constituents in gastroids and gastric epithelial cells. Gut $\mathbf{6 4}$ 720-730 (2015).

52. Huang, J. Y. et al. Chemodetection and destruction of host urea allows Helicobacter pylori to locate the epithelium. Cell Host Microbe 18, 147-156 (2015).

53. Holokai, L. et al. Increased programmed death-ligand 1 is an early epithelial cell response to Helicobacter pylori infection. PLoS Pathog. 15, e1007468 (2019).

54. Reissfelder, C. et al. Tumor-specific cytotoxic T lymphocyte activity determines colorectal cancer patient prognosis. J. Clin. Investig. 125, 739-751 (2015)

55. Kao, J. Y. et al. Helicobacter pylori immune escape is mediated by dendritic cell-induced Treg skewing and Th17 suppression in mice. Gastroenterology 138, 1046-1054 (2010).

56. Sebrell, T. A. et al. A novel gastric spheroid co-culture model reveals chemokine-dependent recruitment of human dendritic cells to the gastric epithelium. Cell. Mol. Gastroenterol. Hepatol. 8, 157-171 (2019).

57. Powell, D. W., Pinchuk, I. V., Saada, J. I., Chen, X. \& Mifflin, R. C. Mesenchymal cells of the intestinal lamina propria. Annu Rev. Physiol. 73, 213-237 (2011).

58. Roulis, M. \& Flavell, R. A. Fibroblasts and myofibroblasts of the intestinal lamina propria in physiology and disease. Differentiation 92, 116-131 (2016).

59. Meran, L., Baulies, A. \& Li, V. S. W. Intestinal stem cell niche: the extracellular matrix and cellular components. Stem Cells Int. 2017, 7970385 (2017).

60. Yoo, B. B. \& Mazmanian, S. K. The enteric network: interactions between the immune and nervous systems of the gut. Immunity 46, 910-926 (2017).

61. Gentile, M. E. \& King, I. L. Blood and guts: the intestinal vasculature during health and helminth infection. PLoS Pathog. 14, e1007045 (2018).

62. Almeqdadi, M., Mana, M. D., Roper, J. \& Yilmaz, O. H. Gut organoids: minitissues in culture to study intestinal physiology and disease. Am. J. Physiol. Cell Physiol. 317, C405-C419 (2019).

63. Fritsch, C. et al. Epimorphin expression in intestinal myofibroblasts induces epithelial morphogenesis. J. Clin. Invest. 110, 1629-1641 (2002).

64. Yeung, T. M., Chia, L. A., Kosinski, C. M. \& Kuo, C. J. Regulation of self-renewal and differentiation by the intestinal stem cell niche. Cell Mol. Life Sci. $\mathbf{6 8}$ 2513-2523 (2011).

65. Lahar, N. et al. Intestinal subepithelial myofibroblasts support in vitro and in vivo growth of human small intestinal epithelium. PLOS ONE 6, e26898 (2011).

66. Lei, N. Y. et al. Intestinal subepithelial myofibroblasts support the growth of intestinal epithelial stem cells. PLOS ONE 9, e84651 (2014).

67. Jabaji, Z. et al. Use of collagen gel as an alternative extracellular matrix for the in vitro and in vivo growth of murine small intestinal epithelium. Tissue Eng. Part C. Methods 19, 961-969 (2013).

68. Jabaji, Z. et al. Type I collagen as an extracellular matrix for the in vitro growth of human small intestinal epithelium. PLOS ONE 9, e107814 (2014).

69. Grundy, D. \& Schemann, M. Enteric nervous system. Curr. Opin. Gastroenterol. 22, 102-110 (2006)

70. Mahe, M. M. Engineering a second brain in a dish. Brain Res. 1693, 165-168 (2018).

71. Fattahi, F. et al. Deriving human ENS lineages for cell therapy and drug discovery in Hirschsprung disease. Nature 531, 105-109 (2016).

72. Workman, M. J. et al. Engineered human pluripotent-stem-cell-derived intestinal tissues with a functional enteric nervous system. Nat. Med. 23 49-59 (2017).

73. Uchida, $\mathrm{H}$. et al. A xenogeneic-free system generating functional human gut organoids from pluripotent stem cells. JCl Insight 2, e86492 (2017).

74. Macdonald, T. T. \& Monteleone, G. Immunity, inflammation, and allergy in the gut. Science 307, 1920-1925 (2005).

75. Santaolalla, R., Fukata, M. \& Abreu, M. T. Innate immunity in the small intestine. Curr. Opin. Gastroenterol. 27, 125-131 (2011).

76. Agace, W. W. \& McCoy, K. D. Regionalized development and maintenance of the intestinal adaptive immune landscape. Immunity 46, 532-548 (2017).

77. Allaire, J. M. et al. The intestinal epithelium: central coordinator of mucosal immunity. Trends Immunol. 39, 677-696 (2018).
78. Noel, G. et al. A primary human macrophage-enteroid co-culture model to investigate mucosal gut physiology and host-pathogen interactions. Sci. Rep. 7, 45270 (2017).

79. Lindemans, C. A. et al. Interleukin-22 promotes intestinal-stem-cell-mediated epithelial regeneration. Nature 528, 560-564 (2015).

80. Jung, K. B. et al. Interleukin-2 induces the in vitro maturation of human pluripotent stem cell-derived intestinal organoids. Nat. Commun. 9, 3039 (2018).

81. Kasendra, M. et al. Development of a primary human small intestine-on-achip using biopsy-derived organoids. Sci. Rep. 8, 2871 (2018).

82. Watson, C. L. et al. An in vivo model of human small intestine using pluripotent stem cells. Nat. Med. 20, 1310-1314 (2014).

83. Norman, J. M., Handley, S. A. \& Virgin, H. W. Kingdom-agnostic metagenomics and the importance of complete characterization of enteric microbial communities. Gastroenterology 146, 1459-1469 (2014)

84. Gagliardi, A. et al. Rebuilding the gut microbiota ecosystem. Int J. Environ. Res. Public Health 15, 1679 (2018).

85. Thursby, E. \& Juge, N. Introduction to the human gut microbiota. Biochem J. 474, 1823-1836 (2017).

86. O'Hara, A. M. \& Shanahan, F. The gut flora as a forgotten organ. EMBO Rep. 7 688-693 (2006).

87. Shaffiey, S. A. et al. Intestinal stem cell growth and differentiation on a tubular scaffold with evaluation in small and large animals. Regen. Med. 11, 45-61 (2016).

88. Hou, Q. et al. Lactobacillus accelerates ISCs regeneration to protect the integrity of intestinal mucosa through activation of STAT3 signaling pathway induced by LPLs secretion of IL-22. Cell Death Differ. 25, 1657-1670 (2018).

89. Hill, D. R. et al. Bacterial colonization stimulates a complex physiological response in the immature human intestinal epithelium. Elife $\mathbf{6}$, https://doi. org/10.7554/eLife.29132 (2017).

90. VanDussen, K. L. et al. Development of an enhanced human gastrointestinal epithelial culture system to facilitate patient-based assays. Gut 64, 911-920 (2015)

91. Karve, S. S, Pradhan, S, Ward, D. V. \& Weiss, A. A Intestinal organoids model human responses to infection by commensal and Shiga toxin producing Escherichia coli. PLOS ONE 12, e0178966 (2017).

92. Rajan, A. et al. Novel segment- and host-specific patterns of enteroaggregative Escherichia coli adherence to human intestinal enteroids. MBio $\mathbf{9}$ https://doi.org/10.1128/mBio.02419-17 (2018).

93. In, J. et al. Enterohemorrhagic Escherichia coli reduce mucus and intermicrovillar bridges in human stem cell-derived colonoids. Cell Mol. Gastroenterol. Hepatol. 2, 48-62.e43 (2016).

94. Zhang, Y. G., Wu, S., Xia, Y. \& Sun, J. Salmonella-infected crypt-derived intestinal organoid culture system for host-bacterial interactions. Physiol. Rep. 2, e12147 (2014)

95. Forbester, J. L. et al. Interaction of Salmonella enterica Serovar Typhimurium with intestinal organoids derived from human induced pluripotent stem cells. Infect. Immun. 83, 2926-2934 (2015).

96. Wilson, S. S., Tocchi, A., Holly, M. K., Parks, W. C. \& Smith, J. G. A small intestinal organoid model of non-invasive enteric pathogen-epithelial cell interactions. Mucosal Immunol. 8, 352-361 (2015).

97. Leslie, J. L. et al. Persistence and toxin production by Clostridium difficile within human intestinal organoids result in disruption of epithelial paracellular barrier function. Infect. Immun. 83, 138-145 (2015).

98. Heo, I. et al. Modelling Cryptosporidium infection in human small intestinal and lung organoids. Nat. Microbio/ 3, 814-823 (2018).

99. Finkbeiner, S. R. et al. Stem cell-derived human intestinal organoids as an infection model for rotaviruses. MBio 3, e00159-00112 (2012).

100. Saxena, K. et al. Human intestinal enteroids: a new model to study human rotavirus infection, host restriction, and pathophysiology. J. Virol. 90, 43-56 (2016).

101. Yin, Y. et al. Modeling rotavirus infection and antiviral therapy using primary intestinal organoids. Antivir. Res. 123, 120-131 (2015).

102. Ettayebi, K. et al. Replication of human noroviruses in stem cell-derived human enteroids. Science 353, 1387-1393 (2016).

103. Sarker, M., Naghieh, S., Sharma, N. \& Chen, X. 3D biofabrication of vascular networks for tissue regeneration: a report on recent advances. J. Pharm. Anal. 8, 277-296 (2018)

104. Nashimoto, Y. et al. Integrating perfusable vascular networks with a threedimensional tissue in a microfluidic device. Integr. Biol. 9, 506-518 (2017). 
105. Park, S. E., Georgescu, A. \& Huh, D. Organoids-on-a-chip. Science 364, 960-965 (2019).

106. Liu, H. et al. Advances in hydrogels in organoids and organs-on-a-chip. Adv. Mater. 31, 1902042 (2019)

107. Kratochvil, M. J. et al. Engineered materials for organoid systems. Nat. Rev. Mater. 4, 606-622 (2019).

108. Jung, C. S., Kim, B. K., Lee, J., Min, B.-H. \& Park, S.-H. Development of printable natural cartilage matrix bioink for 3D printing of irregular tissue shape. Tissue Eng. Regenerative Med. 15, 155-162 (2018).

109. Zhuang, P., Sun, A. X., An, J., Chua, C. K. \& Chew, S. Y. 3D neural tissue models: from spheroids to bioprinting. Biomaterials 154, 113-133 (2018).

110. Yamaoka, N. et al. Open-chamber co-culture microdevices for single-cell analysis of skeletal muscle myotubes and motor neurons witneuromuscular junctions. BioChip J. 13, 127-132 (2019).

111. Jin, Y. et al. Three-dimensional brain-like microenvironments facilitate the direct reprogramming of fibroblasts into therapeutic neurons. Nat. Biomed. Eng. 2, 522-539 (2018).
112. Xiang, Y. et al. Fusion of regionally specified hPSC-derived organoids models human brain development and interneuron migration. Cell Stem Cell 21, 383-398.e387 (2017).

113. Jin, Y. et al. Vascularized liver organoids generated using induced hepatic tissue and dynamic liver-specific microenvironment as a drug testing platform. Adv. Funct. Mater. 28, 1801954 (2018).

114. Koike, $H$. et al. Modelling human hepato-biliary-pancreatic organogenesis from the foregut-midgut boundary. Nature 574,1-5 (2019).

115. Tripathi, A. et al. The gut-liver axis and the intersection with the microbiome Nat. Rev. Gastroenterol. Hepatol. 15, 397 (2018).

116. Carabotti, M., Scirocco, A., Maselli, M. A. \& Severi, C. The gut-brain axis: interactions between enteric microbiota, central and enteric nervous systems. Ann. Gastroenterol. 28, 203 (2015).

117. Hsiao, E. Y. Gastrointestinal issues in autism spectrum disorder. Harv. Rev. Psychiatry 22, 104-111 (2014).

118. Sullivan, P. B. Gastrointestinal disorders in children with neurodevelopmental disabilities. Dev. Disabilities Res. Rev. 14, 128-136 (2008). 